\title{
MCA-MAC: Modified Cooperative Access MAC Protocol in Wireless Sensor Networks
}

\author{
Aya Hossam ${ }^{1}$, Tarek Salem², Anar Abdel Hady², and Sherine Abd El-Kader ${ }^{2}$ \\ ${ }^{1}$ Electrical Engineering Department, Faculty of Engineering (Shoubra), Benha University, Egypt \\ ${ }^{2}$ Computers and Systems Department, Electronics Research Institute, Egypt
}

\begin{abstract}
Throughput, energy efficiency and average packet delivery delay are some of the most crucial metrics that should be considered in Wireless Sensor Networks (WSNs). This paper proposes a modified Medium Access Control (MAC) protocol for WSNs, called (MCA-MAC). MCA-MAC aims to improve the previous metrics and thus the overall performance of WSNs through using cooperative communication. It enables source nodes from using intermediate nodes as relays to send their data through them to the access point. MCA-MAC protocol is also acting as a cross layer protocol where the best end-to-end path between the source and destination is found through an efficient algorithm. Mathematical analysis demonstrates that MCA$M A C$ protocol can determine the optimal relay node that has the minimum transmission time for the given source-destination pair. Using Multi-Paradigm Programming Language (MATLAB) simulation environment, this paper estimates MCA-MAC protocol performance in terms of system throughput, energy efficiency and delay. The results show that MCA-MAC protocol outperforms the existing scheme called Throughput and Energy aware Cooperative MAC protocol (TEC-MAC) protocol under ideal and dynamic channel conditions. Under ideal conditions, MCA-MAC protocol achieved throughput, and energy efficiency improvements of $12 \%$, and $50 \%$ respectively, more than TEC-MAC protocol. While the packet delay through using MCA-MAC has been decreased by about $48 \%$ less than TEC-MAC protocol.
\end{abstract}

Keywords: WSN, MAC protocol, cooperative communication, energy efficient.

Received April 12, 2020; accepted September 7, 2020

https://doi.org/10.34028/iajit/18/3/9

\section{Introduction}

Wireless Sensor Network (WSN) is lately considered as one of the most important breakthroughs in technology due to its high demand in various applications. Recent manufactures of sensor networks target making it available at low cost to end users in daily applications. In addition, the features of real time, accurate information and ease of deployment make WSNs appealing to various application fields such as military [11], medical [9], commercial and agricultural $[4,5]$ applications. However, there are several challenges that need to be addressed by designers of WSNs to overcome the shortcomings during the implementation of them. The wireless environment itself comes at the top of these challenges due to the interference, signal loss, reduction in throughput with respect to distance and delay in data delivery that accompany this medium. Thus, improving these metrics is considered a high challenge in WSNs $[6,10$, $12,16,18]$.

On the other hand, minimizing the overall energy consumption is highly desirable challenge to extend the lifetime of WSNs. Data transmission is one of the most energy consumption activities WSN that must be put into account during the design process.

Cooperative communication among sensor nodes helps to reduce the challenges that face WSNs.
Cooperative communication is a term that refers to the utilization of the broadcasting nature of WSNs and the dense distribution of nodes in the area for collaboration of nodes together [2, 3]. In cooperative communication, relay node(s) are used by source nodes to route data to their destinations and thus it reduces the transmission delay and energy used for reaching a successful transmission [1, 11, 17, 19]. That is why cooperative communication technique is an emerging technology in improving the performance of sensor networks. Multiple-relay [1] and single-relay [19] strategies are the basic two approaches for cooperative communication. In multiple-relay strategy, the implementation complexity and the cooperation overhead are relatively higher than that of single-relay strategy. According to that, the single-relay cooperative strategy is practically considered much suitable for WSNs with resource-constrained.

This paper aims to develop and design a cooperative MAC protocol to improve WSN performance in terms of throughput, energy efficiency, longer lifetime and quick data delivery. In this paper, a cross layer MAC protocol, called Modified Cooperative Access MAC (MCA-MAC) protocol has been proposed for WSNs. This protocol proposes a cross layer technique involving both the MAC and physical layer. In MCAMAC, data transmission algorithm considers both the data rate of source nodes and wireless channel 
conditions at the moment of transmission. Using the MAC and physical layer available information MCAMAC protocol will choose adaptively between direct transmission mode and cooperative transmission mode for data packets. By using both methods adaptively this cooperative method improves the overall performance of WSNs. In non-cooperative (i.e., direct transmission) mode, data is transmitted directly from the source to the destination, while in the cooperative mode a relay node is chosen from a list of neighboring candidates.

The proposed MCA-MAC protocol for WSN presents novel contributions outlined below:

1. A developed relay node selection algorithm is introduced to select the relay node with the minimum transmission time to route data of the source node to the destination.

2. Less control packets and overhead.

3. Development of a mathematical model to evaluate the performance of MCA-MAC protocol under ideal and imperfect wireless channel conditions. This analytical model considers the multi-rate, channel conditions, cooperative transmission, and saturated traffic load.

The following sections in this paper are arranged as follows: section 2 introduces a brief literature review on the related works. Section 3 elaborates the proposed system description. Section 4 explains the operation of the proposed MCA-MAC protocol. Section 5 presents the analytical model of the proposed MCA-MAC protocol. Section 6 discusses the simulation results through a comparative analysis. In the end, the conclusions and future work are presented in section 7.

\section{Related Work}

Several cooperative techniques have been proposed in many layers such as the physical layer, MAC layer and even through multiple layers. Mainaud et al. [14] aim to fill the gap between cooperative communications technique developed for physical layer and an appropriate MAC layer scheme for WSN. They introduced a new MAC layer scheme named Wireless Sensor Cooperative-MAC (WSC-MAC) protocol to enhance the overall reliability of the network by using cooperative communication. In WSC-MAC, each node maintains a link state table that stores the link quality between neighboring nodes. The rely node selection is based on that the link quality between itself and the access point must be better than the direct sourcedestination link. Liu et al. [13] proposed a node cooperation mechanism. In this mechanism, one or multiple nodes with higher channel gain and enough residual energy can help a sender to relay its data packets to its destination. First, they suggested a transmission power optimization algorithm in order to increase lifetime and improve the energy efficiency. This was done by optimizing the transmission powers of the source node and its cooperative relay nodes to increase their minimum residual energy after their data packet transmissions. This paper designed a cooperative relay node contention mechanism to utilize the ability of the source node to effectively select a group of relays with the lowest energy consumption and the best channel quality for cooperative transmissions, thus further improving the energy efficiency. On the other hand, Nacef et al. [15] proposed another cooperative MAC protocol called COSMIC for WSNs. In this protocol, the retransmissions were triggered by the destination after an erroneous packet reception. The relays in the network were enabled to forward the original packets to the destination node, as ARQ defines, using better channel conditions in terms of Packet Error Rate (PER).

A Busy Tone Based Cooperative MAC Protocol (BTAC) [17] and Throughput and Energy aware Cooperative MAC protocol (TEC-MAC) [11] are two similar cooperative MAC protocols for WSNs that take advantage of the multi-rate capability of the IEEE 802.11 [12] in which high-data-rate nodes assist lowdata-rate nodes to transmit data. In these two protocols, each node promiscuously listens to ongoing transmissions to establish and maintain a relay list or table which waste a lot of time. The main difference between BTAC and TEC-MAC is that the relay node in TEC-MAC can forward its own data packet to destination after sending source's data packet.

In this paper, the proposed MCA-MAC overcomes the problems encountered by BTAC and TEC-MAC; as the relay node is selected using a different distributed approach and does not need large overheads. The relay can be chosen dynamically based on an evaluated metric value. In MAC-MAC, the node with the least end-to-end delay is chosen as a relay node from the set of neighboring nodes to route data of the source node to the destination.

\section{System Description}

This paper considers a wireless sensor network based on IEEE 802.11b [7, 12, 20] standard which provides multi transmission data rates of $11,5.5,2$, and 1 Mbps. In this paper, a large WSN of 150 static nodes was assumed with uniformly distributed nodes. A single physical wireless channel has been used for data transmission. Also, a slow fading channel has been adopted to keep the channel conditions unchanged through the MAC frame transmission. This paper uses also a non-variable transmission power by all nodes.

The Access Point (AP) listens to and receives both the source and relay node signals according to the broadcast nature of wireless channels. There are two data transmission modes which are direct transmission mode and single-relay cooperative mode. In the direct transmission mode, data is sent directly from the 
source to the AP as the transmission process involves only the source and destination nodes. While the single-relay cooperative mode involves two phases; the first phase involves the selection of a relay node by the source node when it has data to send. Source node chooses the node that has the minimal transmission time from its neighbors to the AP. In the second phase, the source sends its data "DATA-S" to the relay node which in turn sends it to the AP and afterwards it sends its own data to the AP. The two modes are shown in Figure 1.

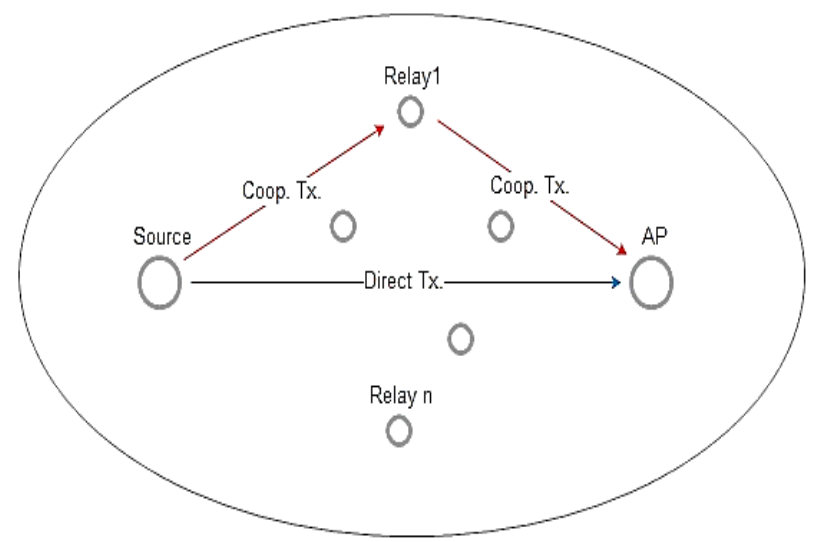

Figure 1. The system model.

\section{Operation of Proposed MCA-MAC Protocol}

In the proposed protocol, each data transmission consists of two parts: control and data. The control part determines the data transmission mode, including the relay selection, and the cross-layer data transmission algorithms. While based on the transmission mode the data part is used for sending, receiving, or forwarding data packets. In this section the relay selection and data transmission algorithms are elaborated in detail.

\subsection{Relay Selection Algorithm}

Using opportunistic relaying, the relay node with the least end-to-end delay is chosen from the set of neighboring nodes $M$. The source node calculates its transmission data rate $\left(\mathrm{R}_{\mathrm{sd}}\right)$ to the AP based on channel quality between them. And similarly, it can figure out the achievable data rate $\left(R_{\text {sr }}\right)$ from it to its set of neighboring nodes and also their data rate $\left(\mathrm{R}_{\mathrm{rd}}\right)$ to the AP by overhearing the channel during their data transmission. According to [2], data transmission time for direct transmission can be calculated as follows:

$$
T_{d}=\frac{8 L}{R_{S d}}
$$

Where $L$ is the packet length. On the other hand, the data transmission time of cooperative transmission for a source node (i) can be calculated as the sum of the time consumed in transmission from the source to the relay node and the time of transmission from the relay node to the AP. The equation for calculating the transmission time for cooperative transmission is given as follows:

$$
T_{c, i}=\frac{8 L}{R_{s r}}+\frac{8 L}{R_{r d}}
$$

For a neighbor relay node $\mathrm{j}$, it must satisfy the condition of $T_{c, i}<T_{d}$ to become an elected relay node. The elected relay node that achieves the minimum transmission time from the source to the AP becomes the best relay node for this source-AP pair.

In order to select the relay node with the minimum transmission time in cooperative transmission the following Algorithm is used:

- At the beginning phase, neighbouring nodes start a timer $T_{r}$ with the value of the parameter $T_{c, r}$.

- The timer $\left(T_{r}\right)$ with the minimal value of $T_{c, r}$ expires first.

- The node with the timer that expires sends an Ready To Help (RTH) packet to inform the source of its ability to serve as its relay node.

- When the rest of the neighbouring nodes overhear the RTH packet, they in turn stop their timers and understand that the best relay node has been chosen.

- The node that sent the RTH becomes the best relay node for this source node and will be ready for the cooperative transmission.

\subsection{MCA-MAC Data Transmission Algorithm}

MCA-MAC protocol works in one of two transmission modes: RTS/CTS direct transmission mode and singlerelay cooperative transmission mode. Figure 2 describes the access mechanism of MCA-MAC protocol due to the control packets handshake Figure 2-a) and the data packets Figure 2-b).

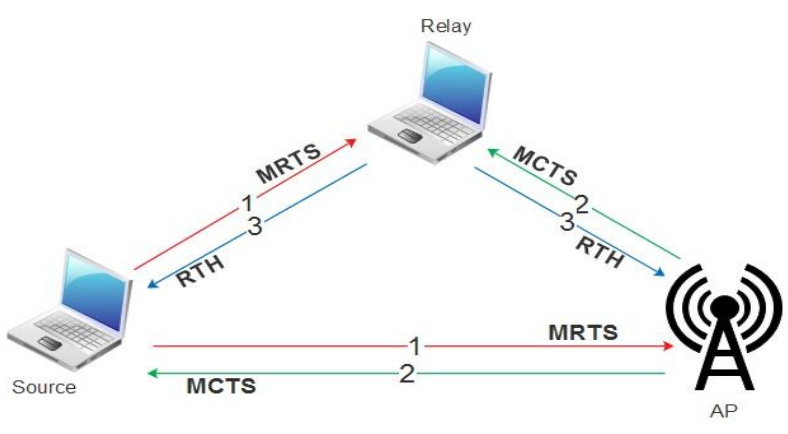

a) Control packets handshake.

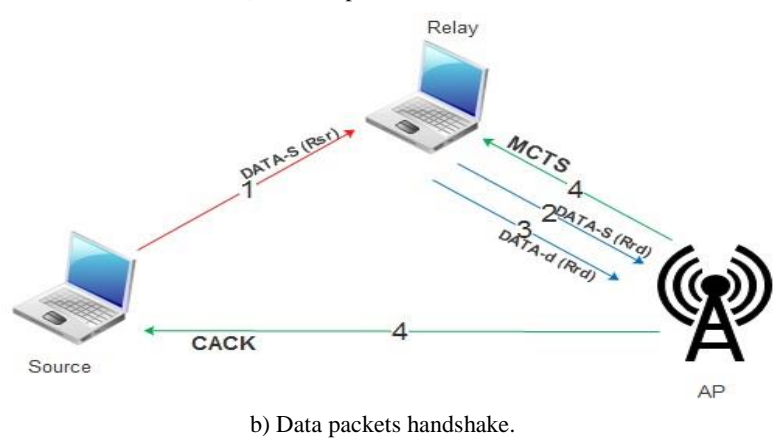

Figure 2. The access mechanism of MCA-MAC protocol. 


\subsubsection{Single-Relay Cooperative Transmission Mode}

This scheme is done for large frame lengths only. The source node sends its data frame to AP through a relay node. When the source node has a large data frame (larger than RTS threshold) to send, it waits for a random back-off time and sends a Modified Ready To Send (MRTS) packet to the AP. After receiving the MRTS packet, the AP waits for Short Inter-Frame Space time (SIFS) and replies with a Modified Clear To Send (MCTS) packet waits for RTH packet from neighbor nodes. When the relay node is available, it sends a RTH packet to source and AP. The source node transmits its data packet with data rate $R_{\mathrm{sr}}$ to the relay node only. The relay node transmits its own data packet with data rate $\mathrm{R}_{\mathrm{rd}}$ to the AP at the same time of receiving data packet of source node. Finally, after receiving data packets successfully from both source and relay, the AP replies with Cooperative Acknowledgement (CACK) to both source and relay. In case of the relay node is not available, the source node will use the direct transmission scheme. Figure 3 shows the steps of data transmission using single relay cooperative mode.

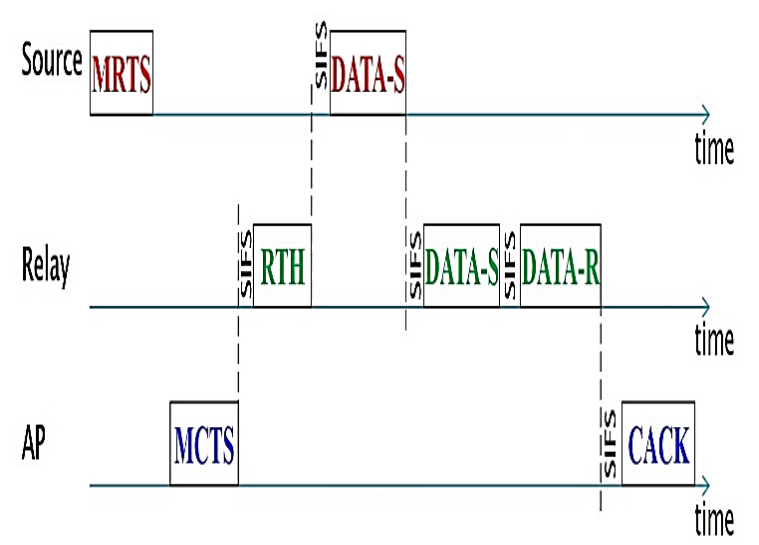

Figure 3. Single-relay cooperative transmission mode.

\subsubsection{Direct Transmission Mode}

This mode is used either when the relay node is unavailable or when the data packet is less than the RTS threshold. As mentioned in the single-relay cooperative transmission mode, when the source node has a large data frame to send, it sends an MRTS packet to the AP and relay nodes. The AP replies with an MCTS packet to the source and relay nodes. After the source node waits the SIFS time and does not receive an RTH packet from the relay node which indicates it's not available, the source sends its data directly to the AP. The AP replies with an Acknowledgement (ACK) packet to the source node. In case the data frame length of the source is less than the RTS threshold, it sends its data packet directly to the AP by the RTS/CTS scheme of IEEE 802.11 DCF [12]. Figure 4 shows the steps of direct transmission mode.

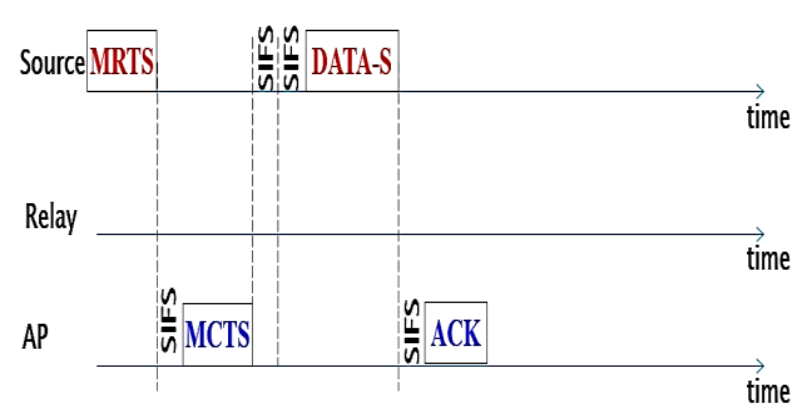

Figure 4. The direct transmission mode.

\section{Analytical Model}

This section presents the analytical model for evaluating MCA-MAC protocol and the average delay performance will be evaluated.

\subsection{Markov Chain Model}

The Markov chain chosen for MCA-MAC protocol is an extension to Bianchi's chain proposed in [20]. It is a two-dimensional chain model that considers frame retry limits. There are two parameters that describe the state of a node in IEEE $802.11 \mathrm{~b}$ which are the backoff stage "i" and the backoff counter value " $k$ ". At the first attempt to send a packet " $\mathrm{i}$ " is initiated by 0 and increased by 1 every time a collision occurs to a maximum value of $\mathrm{m}$. While " $\mathrm{k}$ " is in initiated by choosing a value randomly between $\left[0, W_{i}-1\right]$ where $W_{i}$ is the counter range. $\tau \mathrm{i}$ is the probability that node $\mathrm{i}$ sends data in a random slot of time. When the backoff counter reaches 0 the node can access the medium regardless the backoff stage value reached what. In Markov chain model not only the collision probability is considered, but also the probability of error. Markov chain parameters and the probability of errors are discussed in detail and calculated in [11, 18].Thus for the back off stage, we have:

$$
W_{j}= \begin{cases}2^{j} W_{o}, & j \leq m \\ 2^{m} W_{o}, & j \geq m\end{cases}
$$

In the following subsections, the expressions for the saturated throughput, energy efficiency and delay are derived for MCA-MAC protocol.

\subsection{Saturated Throughput Expression Derivation}

Account transmission errors are taken into account while deriving an expression for saturated throughput of MCA-MAC protocol. Saturated throughput $\eta$ is the ratio of successfully transmitted payload size to the slot time between two consecutive transmissions [11]. The slot time can be sensed in one of four statuses either idle, busy (due to collision), in a successful transmission or an erroneous transmission because of bad channel conditions. We can express $\eta$ as follows according to the adopted definition [11]: 


$$
\eta=\frac{8 L \sum_{i=1}^{N} P_{s, i}\left(1-P_{e, i}\right)}{E\left[T_{I}\right]+E\left[T_{S}\right]+E\left[T_{C}\right]+E\left[T_{E}\right]}
$$

Where $N$ is the network size, $i=1,2, \ldots, N, E[T I]$, $E[T C], E[T S]$ and $E[T E]$ are the average idle slot duration, the average busy slot duration due to collision, the average successful transmission slot duration, and the average erroneous slot duration due channel conditions respectively. The previous values are calculated as in [11].

\subsection{Energy Efficiency Expression Derivation}

In this subsection an expression for energy efficiency is derived for MCA-MAC protocol network. $\varepsilon$, which refers to the energy efficiency is defined as the ratio of sent packet bits successfully to the overall consumed energy in the network. Nodes consume energy in the following activities: backoff $\mathrm{E}_{\mathrm{B}}^{(\mathrm{i})}$, collision $\mathrm{E}_{\mathrm{C}}^{(\mathrm{i})}$, transmission overhearing $\mathrm{E}_{\mathrm{O}}^{(\mathrm{i})}$, transmission errors $\mathrm{E}_{\mathrm{E}}^{(\mathrm{i})}$ and successful transmission $\mathrm{E}_{\mathrm{S}}^{(\mathrm{i})}$. Nodes enter a sleeping mode to save power while they are not involved in any transmission or receiving process. Where PTX, PRX and PIX stand for the power consumed in transmission, receiving and (sensing or being idle) respectively. Thus, the overall network energy efficiency can be expressed as Equation (5) [11]. The calculation of $\mathrm{E}_{\mathrm{B}}^{(\mathrm{i})}, \mathrm{E}_{\mathrm{C}}^{(\mathrm{i})}, \mathrm{E}_{\mathrm{O}}^{(\mathrm{i})}, \mathrm{E}_{\mathrm{E}}^{(\mathrm{i})}$ and $\mathrm{E}_{\mathrm{S}}^{(\mathrm{i})}$ is explained in [11].

$$
\varepsilon=\frac{8 \mathrm{~L} \sum_{\mathrm{i}=1}^{\mathrm{N}} \mathrm{P}_{\mathrm{s}, \mathrm{i}}\left(1-\mathrm{P}_{\mathrm{e}, \mathrm{i}}\right)}{\sum_{\mathrm{i}=1}^{\mathrm{N}}\left(\mathrm{E}_{\mathrm{B}}^{(\mathrm{i})}+\mathrm{E}_{\mathrm{C}}^{(\mathrm{i}}+\mathrm{E}_{\mathrm{O}}^{(\mathrm{i})}+\mathrm{E}_{\mathrm{E}}^{(\mathrm{i})}+\mathrm{E}_{\mathrm{S}}^{(\mathrm{i})}\right)}
$$

\subsection{Delay Expression Derivation}

Finally, in this subsection an expression for average packet delay is derived [8]. It is defined as the time consumed between a packet at the head of its MAC queue to the time it arrives successfully at the AP due to positive acknowledgement. Let $D_{i}(i=1,2 ., N)$ be a random variable representing the packet delay of node $i$. Accordingly, the average packet delay Avg[Di] can be expressed as follows:

$$
\operatorname{Avg}\left[D_{i}\right]=\operatorname{Avg}\left[D_{b, i}\right]+\operatorname{Avg}\left[D_{c, i}\right]+\operatorname{Avg}\left[D_{o, i}\right]+\operatorname{Avg}\left[D_{s, i}\right]+\operatorname{Avg}\left[D_{e, i}\right]
$$

Where $\operatorname{Avg}\left[D_{b, i}\right]$ the average delay in decreasing the backoff counter, $\operatorname{Avg}\left[D_{c, i}\right]$ the average delay in collision transmissions, $\operatorname{Avg}\left[D_{o, i}\right]$ the average delay in holding the backoff counter while other nodes are transmitting, $\operatorname{Avg}\left[D_{s, i}\right]$ the average delay in a successful transmission, $\operatorname{Avg}\left[D_{e, i}\right]$ the average delay due to an erroneous transmission, $\sum_{x=0}^{j} \frac{W_{x}-1}{2}$ is the average number of backoff slots node $i$ needs to send successfully its data packet after ' $j$ ' re-trials.

Let $\overline{N_{b, i}}$ represent the average total number of time slots during backoff duration, which can be calculated as follows:

$$
\overline{N_{b, i}}=\sum_{j=0}^{m} \frac{P_{u, i}^{j}\left(1-P_{u, i}\right)}{1-P_{u, i}^{m+1}} \sum_{x=0}^{j} \frac{W_{x}-1}{2}, i=1,2, \ldots, N
$$

So, the average delay during decreasing the backoff counter $\operatorname{Avg}\left[D_{b, i}\right]$ can be calculated as:

$$
\operatorname{Avg}\left[D_{b, i}\right]=\sigma \overline{N_{b, i}}
$$

Where $\sigma$ is the slot time duration. Now, let $\overline{N_{u, i}}$ be the number of re-trials that node $i$ makes before delivering its packet correctly to its destination. It can be calculated by:

$$
\overline{N_{u, i}}=\sum_{i=0}^{m} \frac{i P_{u}^{i}\left(1-P_{u}\right)}{1-P_{u}^{m+1}}
$$

Now, let $\overline{N_{c, i}}$ be the average number of re-trials that are attempted for collision in the total number of re-trials. It is given by:

$$
\overline{N_{c, i}}=\overline{N_{u, i}} \frac{P_{c, i}}{P_{u, i}}
$$

So, the average delay due to a collision transmission $A v g\left[D_{c, i}\right]$ can be evaluated as follows:

$$
\operatorname{Avg}\left[D_{c, i}\right]=\overline{\mathrm{N}_{c, i}} T_{c}
$$

Where, $T_{c}$ is the collision duration and can be calculated due to direct transmission as:

$$
\mathrm{T}_{\mathrm{c}}^{\mathrm{d}}=\mathrm{T}_{\mathrm{RTS}}+\mathrm{T}_{\mathrm{CTS}}+\mathrm{T}_{\mathrm{SIFS}}+\mathrm{T}_{\mathrm{DIFS}}+\Delta
$$

And the collision duration due to cooperative transmission scheme can be calculated as:

$$
T_{c}^{c}=T_{M R T S}+T_{M C T S}+T_{\text {SIFS }}+T_{D I F S}+\Delta
$$

Now, the average delay in holding the backoff counter while other nodes are transmitting $A v g\left[D_{o, i}\right]$ can be given by:

$$
\operatorname{Avg}\left[D_{o, i}\right]=E\left[Y_{s}\right] \sum_{i=1}^{N-1} P_{s, i}\left(1-P_{e, i}\right) T_{s}+\left(1-P_{s, i}\right) T_{c}+P_{s, i}
$$

Where, $T_{s}, T_{c}$ Equations (13) and (14), and $T_{e}$ is the successful, collision and an erroneous transmission time. Using direct transmission mode, the successful time $T_{S}^{d}$ is given as:

$$
T_{s}^{d}=T_{R T S}+T_{C T S}+\frac{8 L_{s}}{R_{s d}}+T_{P L C P}+T_{A C K}+3 T_{S I F S}+T_{D I F S}+4 \Delta
$$

Using cooperative transmission mode, the successful time $T_{S}^{C}$ is given as:

$T_{S}^{C}=T_{M R T S}+T_{M C T S}+T_{R T H}+\frac{8 L_{s}}{R_{S r}}+\frac{8\left(L_{s}+L_{r}\right)}{R_{r d}}+3 T_{P L C P}+$

$T_{C A C K}+6 T_{\text {SIFS }}+T_{\text {DIFS }}+7 \Delta$

And,

$$
T_{e, i}=\sum_{i=1}^{N} P_{e i, d} T_{e i, d}+\sum_{i=1}^{N} P_{e i, c} T_{e i, c}
$$

Where, $P_{e i, d}, T_{e i, d}, P_{e i, C}$ and $T_{e i, C}$ are given in Equations $10,11,20$, and 21 , respectively. Let $\mathrm{E}\left[Y_{S}\right]$ be the overall number of slots where the backoff counter of node $i$ holds while other nodes are transmitting and consider $P_{t r}$ as the probability that any other node in the network (other than node $i$ ) sends a packet in the idle slot, it is equal to:

$$
P_{t r}=1-\prod_{i=1}^{N}\left(1-\tau_{i}\right)
$$

Then $\mathrm{E}\left[Y_{S}\right]$ can be calculated as follows:

$$
\mathrm{E}\left[Y_{s}\right]=\overline{N_{b, i}} P_{t r}
$$


The average delay of a successful transmission $A v g\left[D_{s, i}\right]$ can be calculated as:

$$
\operatorname{Avg}\left[D_{s, i}\right]=T_{s}
$$

Finally, the average delay due to an erroneous transmission $\operatorname{Avg}\left[D_{e, i}\right]$ can be calculated as follows:

$$
\operatorname{Avg}\left[D_{e, i}\right]=\sum_{j=1}^{k} \overline{N_{e, i}} T_{e, i}
$$

Where, $\overline{N_{e, i}}$ is the average number of transmission errors occurring in control and data packets depending on the type of data transmission scheme and $k=4$ in direct, $k=7$ in cooperative.

In direct transmission scheme, there will be $\overline{N_{e, 1}}, \overline{N_{e, 2}}, \overline{N_{e, 3}}$, and $\overline{N_{e, 4}}$ which can be defined as the average number of transmission errors occurring in RTS, CTS, DATA, and ACK frames respectively. But, in cooperative transmission scheme, there will be $\overline{N_{e, 1}}, \overline{N_{e, 2}}, \overline{N_{e, 3}}, \ldots ., \overline{N_{e, 7}}$ which can be defined as the average number of transmission errors occurring in MRTS, MCTS, RTH, DATA-S from source to relay, DATA-S from relay to AP, DATA-R from relay to AP and CACK frames respectively. $\overline{N_{e, i}}$ is given by:

$$
\overline{N_{e, i}}=\overline{N_{u, i}} \frac{\left(1-P_{c}\right) w_{i}}{P_{u}}
$$

Therefore, the total average packet delay can be calculated by:

$$
D=\frac{1}{N} \sum_{i=1}^{N} \operatorname{Avg}\left[D_{i}\right]
$$

\section{Results and Discussion}

The proposed protocol MCA-MAC has been evaluated using MATLAB to show the effect of parameters such as, the number of sensor nodes and packet length on the performance of the proposed protocol. Based on the results which are extracted from the simulation program of MCA-MAC protocol and TEC-MAC protocol in terms of average delay and packet delivery ratio, we conclude that, MCA-MAC outperforms TECMAC protocol as described in the results below. Table 1 shows the standard values of the parameters used in the simulation.

Table 1. The simulation parameters.

\begin{tabular}{|c|c|c|c|}
\hline Parameter & Value & Parameter & Value \\
\hline MAC header & 28 bytes & Slot time & $9 \mu \mathrm{sec}$ \\
\hline PHY header & 24 bytes & SIFS & $16 \mu \mathrm{sec}$ \\
\hline RTS & 44 bytes & DIFS & $50 \mu \mathrm{sec}$ \\
\hline CTS & 38 bytes & MRTS & 50 bytes \\
\hline ACK & 38 bytes & MCTS & 38.25 bytes \\
\hline RTH & 38 bytes & CW $_{\min }$ & 15 \\
\hline CACK & 38.25 bytes & CW $_{\max }$ & 1023 \\
\hline
\end{tabular}

The MCA-MAC protocol is evaluated for nodes with different data-rates. We study our protocol under the two following conditions:

a. Impact of Ideal Channel Environment: The saturated throughput for the proposed MAC layer has been illustrated in Figure 5. The $\mathrm{x}$-axis represents the number of nodes in WSN in case of ideal channel environment. As illustrated in Figure 5 , when the area of the network increases, the saturated throughput increased. This increase appears in an exponential form. The main reason for that, is the low data rate for the sensor nodes caused by increasing the number of relay nodes. In addition, the throughput of MCA-MAC protocol begins approximately with the same values of TECMAC protocol until reaching 40 nodes, and in the saturation region it is found that the MCA-MAC performs significantly better than TEC-MAC protocol with a $12 \%$ enhancement.

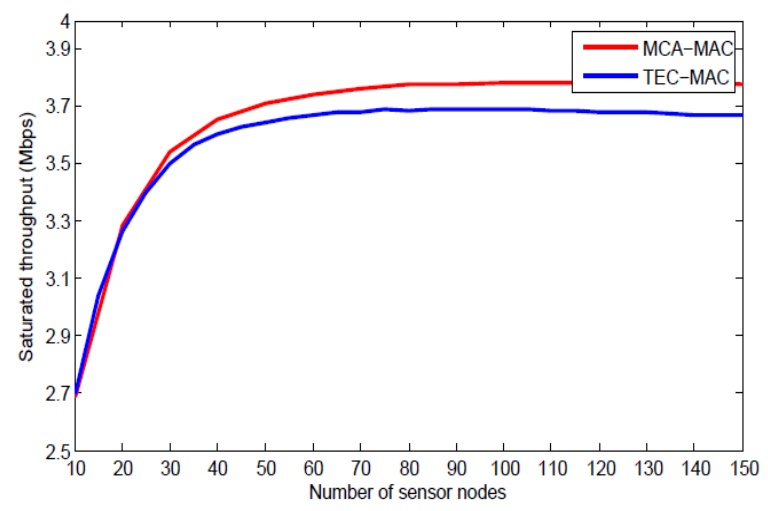

Figure 5. The saturated throughput under different sensor nodes.

Additionally, the saturated throughput of the proposed MAC layer has been shown in Figure 6 but with different packet length. The length of the packet ranges from 400-2000 byte. Those ranges are taken from the standard IEEE $802.11 \mathrm{~b}$ values [12]. As shown in Figure 6, when the length of the packet increases, the saturated throughput increased in both protocols. The main reason is that as the length of the packet increases, the overhead for the protocol is decreased. In average, MCA-MAC protocol outperforms TEC-MAC protocol.

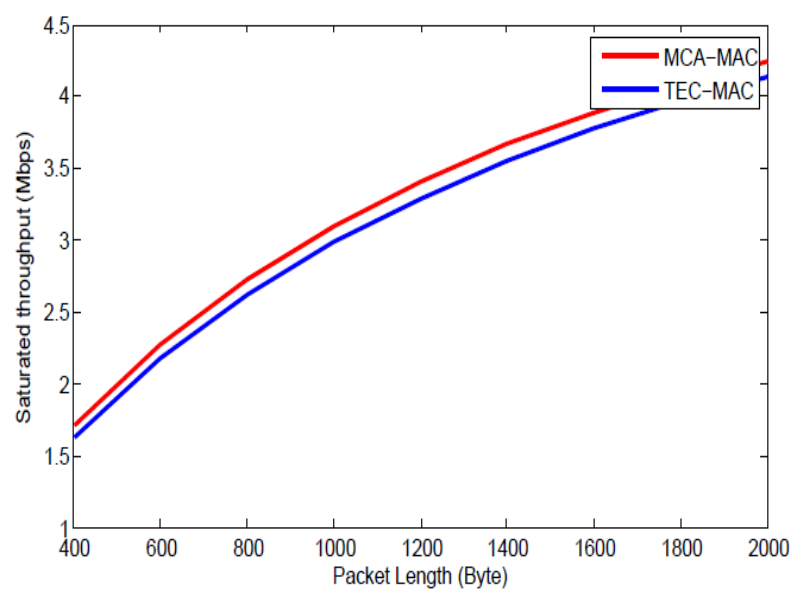

Figure 6. The saturated throughput under different packet length.

In Figure 7, the energy proficiency of the MCAMAC protocol and TEC-MAC protocol are compared with different number of nodes in case of ideal channel environment. It can be concluded that, when the 
number of sensors increases, the energy efficiency for both protocols decreases. This decrease was due to the collision in the environment between nodes. This collision results in sensor nodes retransmission of corrupted packets again and due to that the sensor nodes consume more energy. In general, MCA-MAC protocol achieved a significant improvement reaching $50 \%$ energy saving more than TEC-MAC. This is due to selecting the best relay that saves more time in cooperative transmission. So, it reduces the energy consumption and increase the energy efficiency.

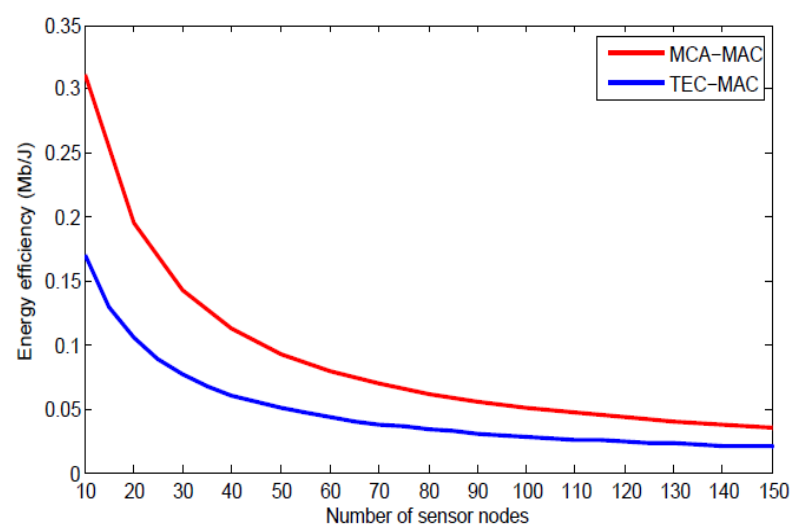

Figure 7. Energy efficiency with different number of sensor nodes.

Figure 8 shows the energy efficiency comparison between MCA-MAC protocol and TEC-MAC protocol with a different $\mathrm{X}$-axis parameter, packet length, in an ideal channel environment. From Figure 8, when the length of the packet increases, the energy efficiency for both MAC protocols decreases. This is because the overhead metric reduces with increasing in the length of the packets which leads to more energy saving. Accordingly, the energy efficiency increased. In average, MCA-MAC protocol outperforms TEC-MAC protocol in energy efficiency of sensor nodes.

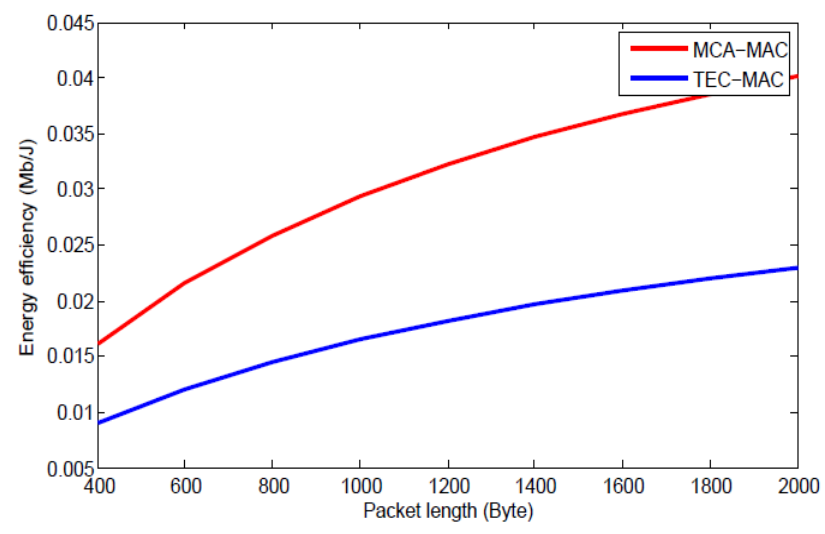

Figure 8. Energy efficiency with different packet length.

In Figure 9, the performance comparison between MCA-MAC protocol and TEC-MAC protocol will be illustrated in terms of the packet delay with different number of sensor nodes with ideal channel environment. As shown in Figure 9, when the number of sensor nodes increases, the packet delay increases for both protocols. That is because of the increase of collision probability due to network size increase. Hence, the packet delay also increases. As shown in Figure 9, the packet delay of MCA-MAC protocol is lower than that of TEC-MAC protocol as the number of sensor nodes increases.

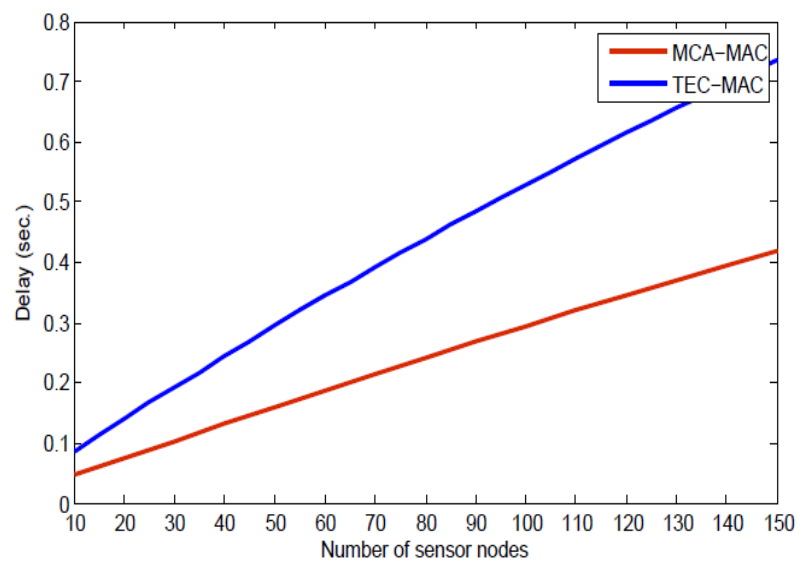

Figure 9. Packet delay under different number of sensors.

In Figure 10, the performance comparison between MCA-MAC protocol and TEC-MAC protocol will be illustrated in terms of the packet delay with different packet length. As shown in Figure 10, when the length of the packet increases, the packet delay for both the protocols increases because as the process of transmission in the network increases the length of the packet increases and accordingly, the packet delay increases. In average, MCA-MAC protocol outperforms TEC-MAC protocol in decreasing packet delay of sensor nodes.

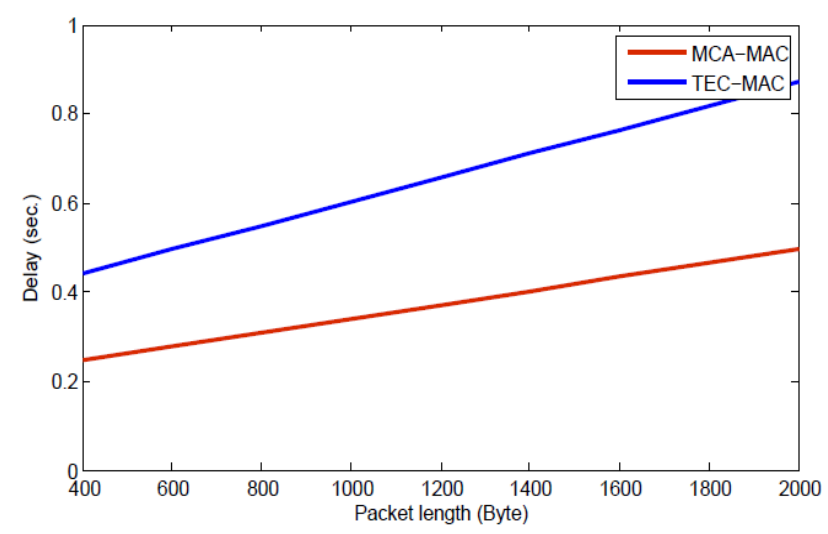

Figure 10. Delay under different packet length.

b. Impact of Dynamic Channel Environment: The saturated throughput for MCA-MAC layer with regards to the number of nodes and packet length has been illustrated in Figures 11 and 12 in case of bit error rate of $1 * 10^{-5}$. As illustrated in Figures 11 and 12, MCA-MAC protocol outperforms TECMAC protocol in achieving more throughput under defective channel environment. In addition, when the number of sensor nodes increases, the saturated throughput for both protocols increases. 


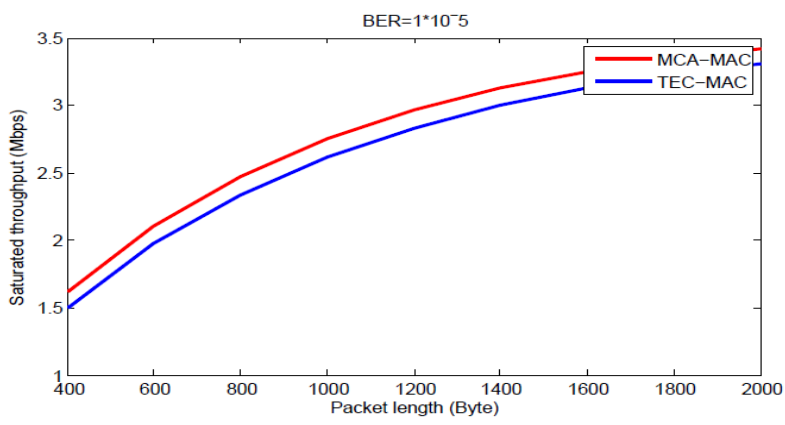

Figure 11. The saturated throughput under different packet length in dynamic channel condition.

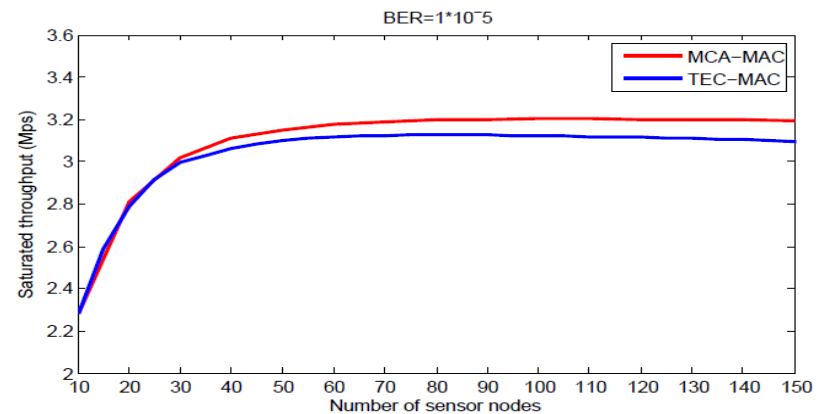

Figure 12. The saturated throughput under different sensor nodes in dynamic channel condition.

The energy efficiency for MCA-MAC protocol and TEC-MAC protocol with regards to the number of nodes and packet length has been illustrated in Figures 13 and 14 , respectively in case of bit error rate $1^{*} 10^{-5}$. As illustrated in Figures 13 and 14, the proposed MAC protocol is better than TEC-MAC protocol in achieving more energy efficiency under defective channel environment.

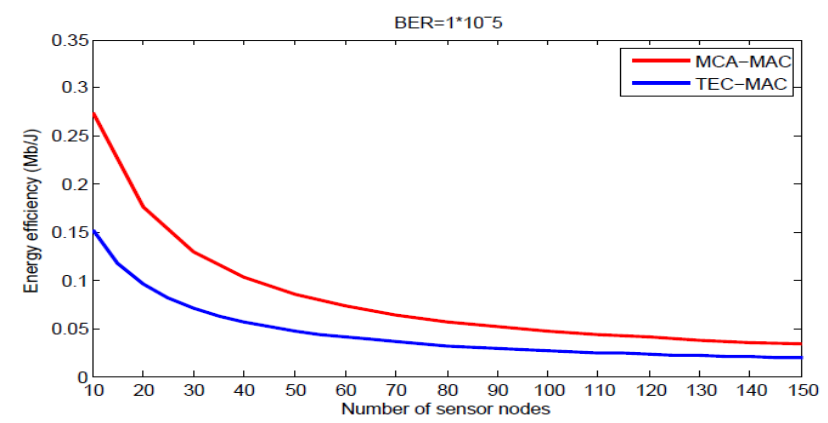

Figure 13. Energy efficiency under different number of sensor nodes in dynamic channel condition.

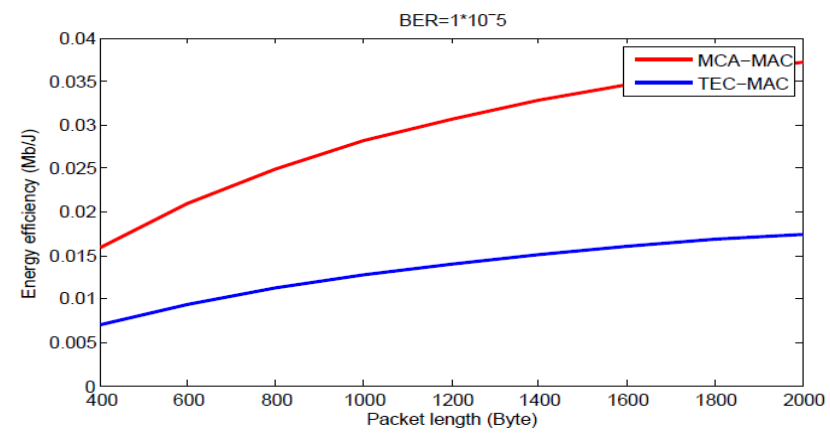

Figure 14. Energy efficiency under different packet length in dynamic channel condition.
Figures 15 and 16 show the packet delay of MCAMAC and TEC-MAC with different number of sensor nodes and length of packet respectively under imperfect channel conditions at Bit Error Rate (BER) of $1 * 10^{-5}$. As shown, the packet delay of the MCAMAC protocol is better compared to TEC-MAC protocol under imperfect channel conditions.

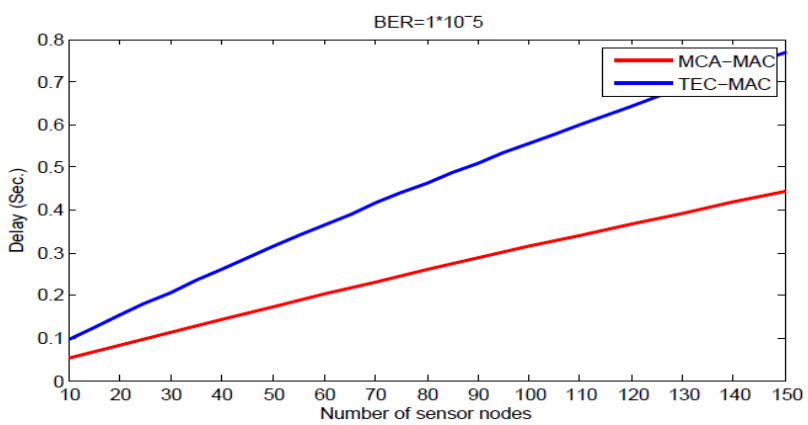

Figure 15. Packet delay under different number of sensor nodes in dynamic channel condition.

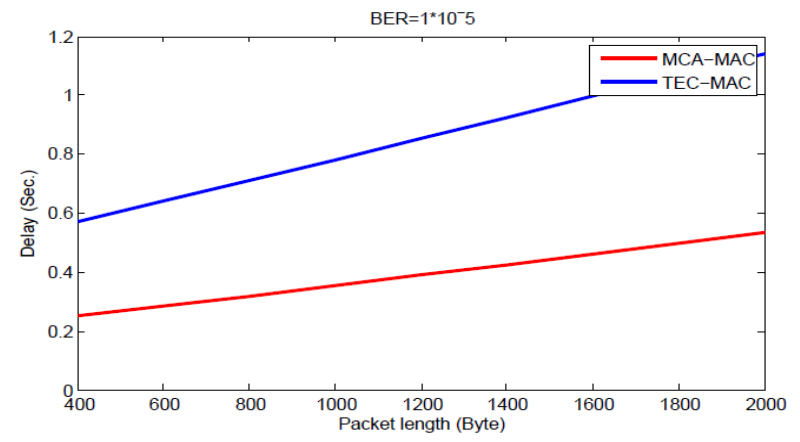

Figure 16. Packet delay under different length of packet in dynamic channel condition.

\section{Conclusions and Future Work}

In this work, a novel MAC layer protocol named MCA-MAC protocol has been proposed for centralized wireless sensor networks. This protocol enhances the network by using the cooperative communication technology where each wireless sensor node uses a relay choice algorithm to discover the most optimal relay in terms of the minimum transmission time. In addition, MCA-MAC protocol proposes a cooperative reliable mechanism, which allows the relay node to send its own data packets without the need of a handshake procedure to access the channel. The proposed protocol is developed to evaluate the performance of MCA-MAC with regards to dynamic and ideal channel environments. The simulation results based on MATLAB demonstrates that the proposed MCA-MAC protocol can meaningfully increase throughput and energy efficiency while decreasing packet delay by about $12 \%, 50 \%, 48 \%$ respectively in comparison to another cooperative MAC protocol called TEC-MAC protocol.

Finally, in the future work it will be interesting to study the system performance of MCA-MAC protocol under real time applications such as medical 
applications. It will also be useful to study the performance of MCA-MAC protocol under unsaturated conditions with more sophisticated traffic models, such as Markov Modulated Poisson Process (MMPP) model.

\section{References}

[1] Bassily R. and Ulukus S., "Deaf Cooperation and Relay Selection Strategies for Secure Communication in Multiple Relay Networks," IEEE Transactions on Signal Processing, vol. 61, no. 6, pp. 1544-1554, 2013.

[2] Cicioğlu M., Bayrakdar M., and Çalhan A., "Performance Analysis of a New MAC Protocol for Wireless Cognitive Radio Networks," Wireless Personal Communications, vol. 108, no. 1, pp. 67-86, 2019.

[3] Bayrakdar M., "Cooperative Communication Based Access Technique for Sensor Networks," International Journal of Electronics, vol. 107, no. 2, pp. 212-225, 2020.

[4] Bayrakdar M., "Employing Sensor Network Based Opportunistic Spectrum Utilization for Agricultural Monitoring," Sustainable Computing: Informatics and Systems, vol. 27, pp. 100404, 2020.

[5] Bayrakdar M., "Energy-Efficient Technique for Monitoring of Agricultural Areas with Terrestrial Wireless Sensor Networks," Journal of Circuits, Systems and Computers, vol. 29, no. 9, pp. 1-17, 2019.

[6] Bayrakdar M., "Exploiting Cognitive Wireless Nodes for Priority-Based Data Communication in Terrestrial Sensor Networks," Electronics and Telecommunications Research Institute Journal, vol. 42, no. 1, pp. 36-45, 2020.

[7] Bianchi G., "Performance Analysis of the IEEE 802.11 Distributed Coordination Function," IEEE Journal on Selected Areas in Communications, vol. 18 , no. 3, pp. 535-547, 2000.

[8] Cheng C. and Tse C., "A Delay-Aware Network Structure for Wireless Sensor Networks with Consecutive Data Collection Processes," IEEE Sensors Journal, vol. 13, no. 6, pp. 2413-2422, 2013.

[9] Gogate U. and Bakal J., "Healthcare Monitoring System Based on Wireless Sensor Network for Cardiac Patients," Biomedical and Pharmacology Journal, vol. 11, no. 3, pp. 1681-1688, 2018.

[10] $\mathrm{He} \mathrm{X}$. and Li F., "Throughput and Energy Efficiency Comparison of One-Hop, Two-Hop, Virtual Relay and Cooperative Retransmission Schemes," in Proceedings of European Wireless Conference, Lucca, pp. 580-587, 2010.

[11] Hossam A., Eldien A., Abdel-Kader S., and El Kader H., "TEC-MAC: Throughput and Energy aware Cooperative MAC Protocol in Wireless
Sensor Networks," International Journal of Computer Science and Applications, vol. 12, no. 4, pp. 55-63 2015.

[12] IEEE 802.11b-1999-IEEE Standard for Information Technology-Telecommunications and information exchange between systemsLocal and Metropolitan networks-Specific requirements-Part 11: Wireless LAN Medium Access Control (MAC) and Physical Layer (PHY) specifications: Higher Speed Physical Layer (PHY) Extension in the $2.4 \mathrm{GHz}$ band, https://standards.ieee.org/standard/802_11b1999.html, Last Visited, 2020.

[13] Liu K., Wu S., Huang B., Liu F., and Xu Z., "A Power-Optimized Cooperative MAC Protocol for Lifetime Extension in Wireless Sensor Networks," Sensors, vol. 16, no. 10, pp. 1630, 2016.

[14] Mainaud B., Gauthier V., and Afifi H., "Cooperative Communication for Wireless Sensors Network: A Mac Protocol Solution Cooperative Communication for Wireless Sensors Network: A Mac Protocol Solution WSC-MAC: A Cooperative Mac Protocol for Wireless Sensors Network," in Proceedings of $1^{\text {st }}$ IFIP Wireless Days, Dubai, pp. 1-5, 2008.

[15] Nacef A., Senouci S., Ghamri-Doudane Y., and Beylot A., "COSMIC: A Cooperative MAC Protocol for WSN with Minimal Control Messages," in Proceedings of $4^{\text {th }}$ IFIP International Conference on New Technologies, Mobility and Security, Paris, pp. 1-5, 2011.

[16] Oralhan Z., Oralhan B., and Yiğit Y., "Smart City Application: Internet of Things (IoT) Technologies Based Smart Waste Collection Using Data Mining Approach and Ant Colony Optimization," The International Arab Journal of Information Technology, vol. 14, no. 4, pp. 423427, 2017.

[17] Sayed S. and Yang Y., "BTAC: A Busy Tone Based Cooperative Mac Protocol for Wireless Local Area Networks," in Proceedings of $3^{\text {rd }}$ International Conference on Communications and Networking in China, Hangzhou, pp. 403409, 2008.

[18] Salem T., Abdel-Mageid S., Abdel-Kader S., and Zaki M., "ICSSSS: An Intelligent Channel Selection Scheme for Cognitive Radio Ad Hoc Networks Using A Self Organized Map Followed By Simple Segregation," Pervasive and Mobile Computing, vol. 39, pp. 195-213, 2017.

[19] Wang D., Liu J., and Yao D., "An EnergyEfficient Distributed Adaptive Cooperative Routing Based on Reinforcement Learning in Wireless Multimedia Sensor Networks," Computer Networks, vol. 178, 2020.

[20] Wu H., Cheng S., Peng Y., Long K., and Ma J., "IEEE 802.11 Distributed Coordination 
Function(DCF): Analysis and enhancement," in Proceedings of IEEE International Conference on Communications, New York, pp. 605-609, 2002,

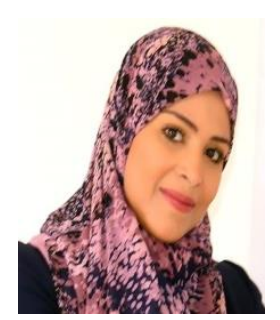

Aya Hossam received the B.S., M.S., Ph.D degree in electronics engineering from Benha University, Cairo, Egypt in 2012, 2015 and 2019 respectively. She is currently an Assistant Professor with the Electrical Engineering Department, electronics branch, Benha University, Cairo, Egypt. Her research interests include IoT, Signal processing, image processing, Acoustic devices, sensor networks and AI. She received the Best Student award from the President, the Best Student award from the Minister of High Education, and the Best Engineer award from President of Egyptian Engineers syndicate.

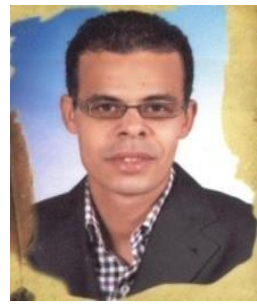

Tarek Salem received his M.S. and Ph.D. in Systems and Computers Engineering from Al-Azhar University in 2012 and 2016, respectively. Since 2016, he is a researcher in Computers and Systems Department at the Electronics Research Institute (ERI) in Egypt. In addition, $\mathrm{He}$ is a Lecturer at Ahram Canadian University (ACU), faculty of computer science from 2018 till now. His research interests include internet of things, Mobile Computing, Cellular Networks, Sensor Networks, Cognitive Radio Networks, and Internet Services and Applications.

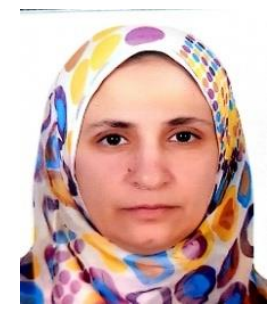

Anar Abdel Hady is a Professional member of IEEE. She received her MSc degree from Faculty of Engineering, Cairo University in 2007. She received her $\mathrm{PhD}$ from Faculty of Engineering, Ain Shams University in 2014. She is currently a researcher in Electronics Research Institute (ERI), Egypt. She was a post doc scholar at Computer Science \& Engineering at Washington University in St Louis, Missouri, USA in 2018-2019. Her research interests are wireless sensor networks, network security and Internet of Things. She is an author of many articles in reputable journals, conferences and book chapters. She was a Co-PI of an accomplished project for developing a prototype of a sensor network for precision agriculture.

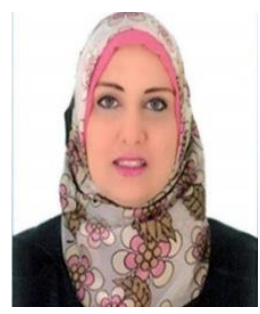

Sherine Abd El-Kader received the M.Sc. and Ph.D. degrees from Faculty of Engineering, Cairo University, in 1998 and 2003 respectively. She is vice president of ERI since 2019. She has been a Professor with the Computers and Systems Department, Electronics Research Institute (ERI), since April 2014. She is a member of the Technological Development and Linkage with Industry and Civil Society Committee, since 2016, and the Head of media committee, since 2017. She is the Head of the Computers and Systems Department, ERI, since 2018. She is also the Head of the Technology, Innovation and Commercialization Office (TICO), since 2018. She is working in many computer networking hot topics such as: the IoT, 5G, cognitive radio, Wi-MAX, Wi-Fi, IP mobility, QoS, wireless sensors Networks, ad-hoc networking, real time traffics, and localization algorithms. 